$56^{\text {ème }}$ Congrès de la SFMBCB, 02010 (2011)

DOI: $10.1051 / \mathrm{sfmbcb} / 20115602010$

(C) Owned by the authors, published by EDP Sciences, 2011

\title{
Apport de la scintigraphie osseuse dans la prise en charge des ostéonécroses secondaires aux bisphosphonates
}

\author{
Agossa $\mathrm{K}^{1}$, Santucci $\mathrm{J}^{1}$, Querellou $\mathrm{S}^{2}$, Boisrame Gastrin $\mathrm{S}^{1}$ \\ ${ }^{1}$ UFR d'Odontologie, Service d'Odontologie, CHRU, Brest, France \\ ${ }^{2}$ UFR de Médecine, Service de Médecine nucléaire, CHRU, Brest, France \\ kevimy.agossa@etudiant.univ-brest.fr
}

\begin{abstract}
Marx et Stern ont été les premiers en 2003 à décrire des cas d'ostéonécroses des maxillaires secondaires aux traitements par bisphosphonates (ONMBP). Ces dernières sont au centre des préoccupations de diverses spécialités, bien au-delà de la sphère buccale. Leur prévalence, la physiopathologie peu connue et le traitement difficile font de cette affection un sujet de préoccupation permanent dans la prise en charge des patients sous biphosphonates (BP). La précocité du diagnostic facilite grandement la thérapeutique. C'est pourquoi les techniques d'imagerie notamment la scintigraphie au $99 \mathrm{~m}$ technetium $\left({ }^{99 \mathrm{~m}} \mathrm{Tc}\right)$ constituent une aide précieuse. L'objectif de cette présentation est de proposer une revue récente de la littérature sur l'apport de la scintigraphie osseuse dans cette pathologie et d'illustrer le sujet par quelques cas cliniques.
\end{abstract}

Couramment employée pour dépister des métastases ostéoblastiques (cancer du sein ou de la prostate), la scintigraphie osseuse est une technique d'imagerie fonctionnelle qui permet de visualiser grâce à l'utilisation d'isotopes radioactifs les remaniements osseux. En effet, l'utilisation d'un bisphosphonate marqué au ${ }^{99 \mathrm{~m}} \mathrm{Tc}$ permet de détecter les zones de forte activité ostéoblastique (Rodan et Fleish 1996). Ces molécules marquées forment des liaisons covalentes avec deux molécules de BP. L'analogie de structure des deux familles de BP implique une similitude dans la distribution et la fixation osseuse selon des mécanismes complexes et mal connus (Paycha et al. 2002). Cette propriété amène à penser que la scintigraphie pourrait servir à détecter les lésions mineures, voire infra-cliniques d'ONMBP qui procéderait également par des mécanismes de remaniement osseux. Les études menées dans ce sens (O’Ryan et al. 2009) bien que peu nombreuses ont donné des résultats intéressants qui placeraient la scintigraphie en tête des méthodes de détection, voire de prédiction des ONMBP.

Toutefois cette grande sensibilité ne suffit pas à faire oublier le manque de spécificité de la technique (Dore et al. 2009). Les hyperfixations en scintigraphie ne signalent que des remaniements osseux importants dont l'étiologie est très variable (inflammation parodontale, métastase, ONMBP). Il serait donc intéressant d'affiner le diagnostic scintigraphique en améliorant le caractère discriminant de la technique. Des possibilités existent (Catalano et al. 2007) par exemple la scintigraphie au MIBI technétié (1-méthoxy isobutyle isonitrile) ou le couplage de la scintigraphie au CT-scan. Des études ultérieures devraient en définir la fiabilité.

La scintigraphie semble être un examen intéressant pour le diagnostic précoce et l'évaluation du traitement des ONMBP. Elle offrirait ainsi des applications à l'oncologue et au chirurgien dentiste, renforçant la prise en charge pluridisciplinaire des patients sous BP. C'est également un vaste sujet de recherche tant dans l'élucidation des mécanismes de fixation que dans l'amélioration du caractère discriminant de la technique. 